\title{
Performance Evaluation of a Hybrid OBS/OCS Network with QoS Differentiation Based on Packet Loss/Delay Requirements
}

\author{
Jordi Perelló, Nuria de Guinea, Salvatore Spadaro, Gabriel Junyent and Jaume Comellas \\ Advanced Broadband Communications Center (CCABA), Universitat Politècnica de Catalunya (UPC) \\ Jordi Girona 1-3, 08034 Barcelona, Spain,e-mail:perello@ac.upc.edu, comellas@tsc.upc.edu
}

\begin{abstract}
This paper introduces and evaluates a hybrid OBS/OCS switch for Quality of Service (QoS) differentiation in next-generation all-optical transport networks. Depending on their QoS requirements, incoming IP packets are collected in delay-sensitive or loss-sensitive assembly queues. Once assembled, those departing delay or losssensitive bursts are sent to the destination over a parallel all-optical hybrid network, where a set of switch ports and wavelengths are dedicated to delay-sensitive OBS-like transmission based on Just Enough Time (JET), whereas the remainder are dedicated to loss-sensitive OCS-like transmission. The obtained results validate the differentiated transport services in the deployed reference network scenario. Moreover, they show that circuit set-up retries are mandatory in OCS to carry loss-sensitive traffic efficiently.
\end{abstract}

Keywords: all-optical networks, hybrid OBS/OCS, QoS differentiation.

\section{INTRODUCTION}

Over the last decade, optical networks have gone through an extensive and rapid evolution [[1]]. Mandated by the explosive growth of Internet users, and the appearance of emerging IP-based bandwidth-consuming services, more and more data capacity has been required to backbone networks. This forced a transition form alreadydeployed voice-centric static networks to flexible IP-centric ones, able to seamlessly support such amounts of heterogeneous data traffic in an efficient way.

In order to overcome the limitations of manual provisioning schemes, a control plane has been introduced in optical transport network, aiming to automatically provision QoS compliant end-to-end optical connections over the transport plane. Such a control plane is typically implemented by means of GMPLS [[2]], a set of protocols defined by the Internet Engineering Task Force (IETF) that cover the required signalling, routing and management functionalities. Indeed, these emerging dynamic Optical Circuit Switching (OCS) networks suppose an important advance on networking evolution. However, due to their coarse granularity (circuits are provisioned on a whole 10 or $40 \mathrm{Gbps}$ wavelength basis) and the inherent two-way signalling overhead, they may behave inefficiently under bursty traffic patterns. In view of this, more advanced optical switching paradigms such as Optical Packet Switching (OPS) and Optical Burst Switching (OBS) have been additionally proposed for mid to long-term optical networks.

In essence, OPS networks [[3]] target at bringing the electronic packet-switching operation directly in the optical domain, which provides a drastically improved adaptation to higher layer dynamics than OCS. Nevertheless, OPS deployment comes up against severe technological limitations [[4]] (e.g., lack of optical RAM memories, infeasible optical header processing or the complex packet-header synchronization at $n s$ timescales'), which leaves OPS realization to a long-term future. In contrast, OBS networks [[5]] become a combination of packet and circuit switched networks, where packets are firstly aggregated in the edge routers and afterwards sent as bursts along bufferless optical networks. This provides the benefit from the statistical multiplexing in the optical domain, allowing better adaptation than OCS to higher layer dynamics. Further, as the header (i.e., the Burst Control Packet, BCP) and the optical burst are transmitted on separated wavelengths, the control information can be electrically processed, which lightens OPS technology requirements.

However, absolute QoS guarantees are still an important yet challenging issue in OBS networks. Furthermore, from an economic point of view, a pure OBS network needs a high number of expensive burst switch ports, which would strongly impact on overall network cost. Supported by these arguments, hybrid OBS/OCS networks have appeared as an efficient and cost-effective solution for future optical transport network infrastructures (e.g., see [[6]][[7]][[8]][[9]]). These networks employ OBS and OCS switching technologies simultaneously. Therefore, it would be the task of the ingress router to choose the most appropriate transport service for the incoming data flow taking, for instance, the flow's QoS requirements or duration into account.

In this paper, we analyze the performance of an OBS/OCS parallel hybrid switch for differentiated QoS transport services based on packet delay/ loss requirements. In particular, an OBS transport is used for delaysensitive data packets, whereas OCS is devoted to the loss-sensitive traffic. This allows us to provide the former kind of traffic with the on-the-fly OBS one-way signalling, as well as the latter one with the assured transmission of the OCS two-way signalling. 


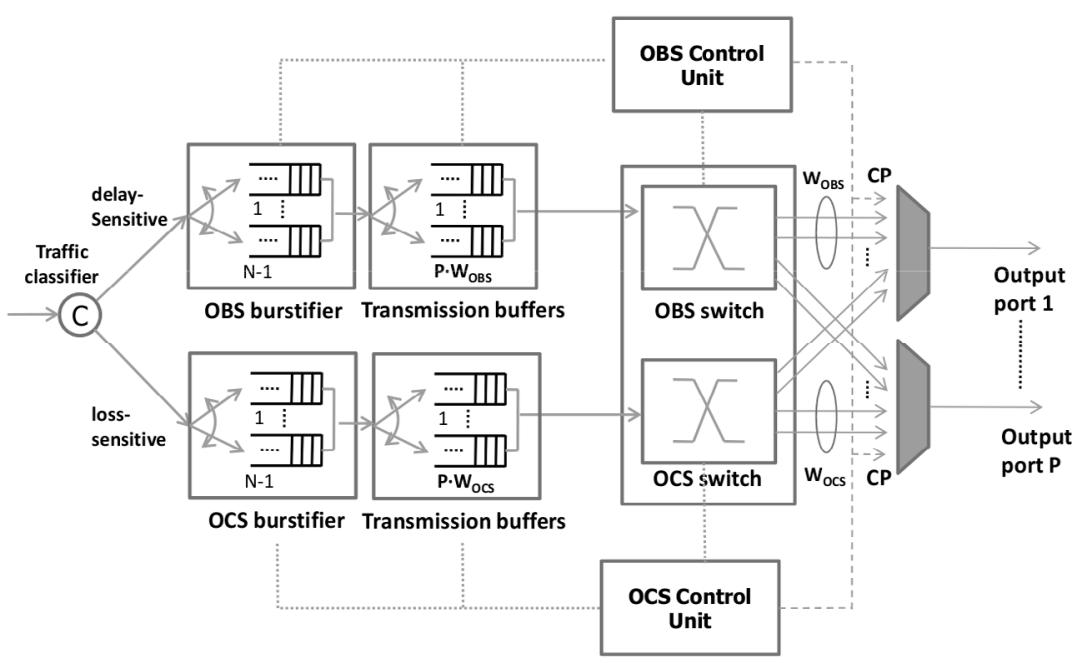

Figure 1. OBS/OCS parallel hybrid switch.

The remainder of the paper continues as follows. Section 2 describes the proposed hybrid OBS/OCS switch architecture. Next, section 3 introduces the scenario where the evaluation has been conducted. The obtained results are presented in section 4 . Finally, section 5 concludes the paper.

\section{OBS/OCS PARALLEL HYBRID SWITCH ARCHITECTURE}

As detailed in [[10]], hybrid OBS/OCS networks can be classified in three different classes depending on the integration between OBS and OCS technologies, namely, client-server, parallel and integrated.

In the client-server class, a DWDM server layer sets up a virtual topology of lightpaths to be used by an OBS client layer on top. Hence, separated networks with separated OBS or OCS equipment are deployed, which may be also owned by different operators. Furthermore, these networks may share no information (overlay model), partial information (augmented model) or complete information (peer model) of the network resources. In the parallel class, the OBS and OCS transport layers are installed side by side over the same network equipment, so that the edge nodes in the network are responsible for selecting the most appropriate transport service for each incoming traffic demand. Therefore, a partitioning of resources exists in the network, dedicated for either OBS or OCS transport. Finally, in the integrated class no resource partitioning is performed in the network but OBS and OCS technologies share the entire set of resources, that is, all network equipment ports are both OBS and OCS switching capable. This makes integrated hybrid OBS/OCS networks optimal in terms of resource utilization, but also the most expensive due to their high technology and control requirements.

Making a good trade-off between network performance and cost, this work focuses on an OBS/OCS parallel hybrid switch, where the use of either OBS or OCS transport is mandated by the incoming traffic QoS requirements. As can be seen in Figure 1, differentiated assembly queues exist in the switch for delay-sensitive and loss-sensitive traffic, one per each of the $N-1$ possible destination nodes. The delay-sensitive queues rely on a timer-based assembly strategy, which provides strict packet queuing time control. Conversely, the losssensitive bursts are assembled following a size-based strategy, ensuring a pre-established burst length.

Once either a delay-sensitive or a loss-sensitive burst is assembled, it is moved to the transmission buffer waiting to be sent through the network. In the proposed switch, one transmission buffer per output wavelength is implemented. Therefore, having the switch $P$ output fibres with $W_{O B S}$ and $W_{O C S}$ wavelengths on each one dedicated to OBS and OCS, respectively, there are $P \cdot W_{O B S}$ transmission buffers for OBS and for $P \cdot W_{O C S}$ for OCS. In order to select the most appropriate transmission buffer (i.e., output wavelength) to queue the assembled burst, the length (i.e., number of queued bursts) of the transmission buffers associated to the preferred output fiber is checked. Following a first-fit selection strategy, if an empty buffer is found, the burst is directly queued there. Conversely, if no empty buffer is found, the burst is queued in the buffer with the lowest length.

Finally, when a burst is ready for transmission (i.e., it reaches the first position in the buffer and the output wavelength is idle) the resource reservation functionality is triggered. In particular, the proposed OBS/OCS parallel hybrid switch implements JET-based one-way reservation [[5]] for the delay-sensitive bursts, which avoids any signalling overhead at the edge node and minimizes the end-to-end latency. This one-way reservation, however, does not assure a successful end-to-end burst transmission, as bursts may be dropped due to contention at intermediate nodes. Aiming to minimize this burst loss probability, an OCS-like two-way reservation is implemented for the loss-sensitive bursts. Although a significantly higher pre-transmission delay is expected in this case, the target here is to fit the packet loss requirements of this kind of traffic. 
The two-way reservation ensures the end-to-end burst transmission provided that the signalling protocol finds the required available resources throughout the path. Nevertheless, this is not always achieved. In case that a NACK message is received at the source edge node informing that no resources have been found on a link composing the route, a certain number or signalling retries are allowed for a loss sensitive-burst before considering it dropped. This number of allowed retries will be conditioned to the maximum allowed latencies for the loss-sensitive traffic and the edge nodes transmission buffer capacities.

\section{SCENARIO UNDER STUDY}

The performance of the proposes OBS/OCS parallel hybrid switch has been evaluated by simulation results on the 9-node network depicted in Fig. 2, where each link carries 8 bidirectional wavelengths operating at $10 \mathrm{Gbps}$. The links have a physical length of $500 \mathrm{~km}$, introducing a propagation delay equal to $2.5 \mathrm{~ms}$. In the network, all nodes are assumed to be OBS/OCS parallel hybrid switches as described in section 2. Specifically, the 8 wavelengths per link are partitioned so that 4 of them are reserved to OBS, while the remaining 4 are used for supporting the OCS-like transmission.

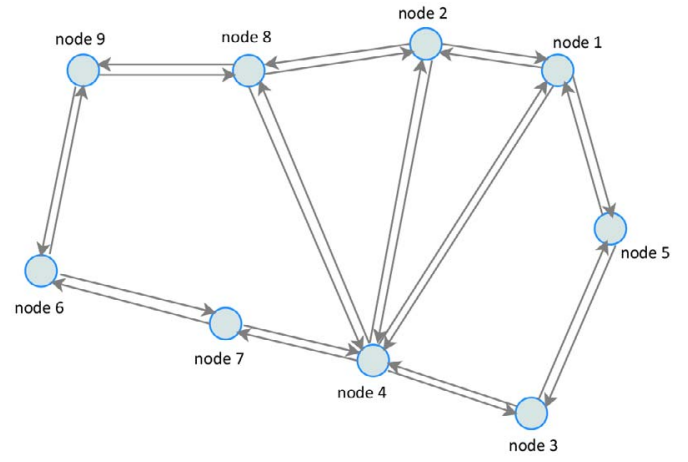

\begin{tabular}{||l|r|}
\hline Number of nodes & 9 \\
\hline Number of fiber links & 12 \\
\hline Mean node degree & 2.67 \\
\hline Mean link length & $500 \mathrm{Km}$ \\
\hline Wavelengths per link & 8 \\
\hline W_OBS & 4 \\
\hline W_OCS & 4 \\
\hline BCP processing time & $10 \mathrm{us}$ \\
\hline OBS matrix switching time & $2.5 \mathrm{us}$ \\
\hline OCS matrix switching time & $1 \mathrm{~ms}$ \\
\hline Packet IAT distribution & neg. exp. \\
\hline Packet length distribution & trimodal \\
\hline t_th (delay-sensitive) & $100 \mathrm{us}$ \\
\hline L_th (loss-sensitive) & $10 \ldots 80$ Mbytes \\
\hline Retries (loss-sensitive) & $1 \ldots 10$ \\
\hline
\end{tabular}

Figure 2. Simulation scenario: network topology (left) and parameters (right).

Regarding the delay-sensitive part of the node, we assume the BCP processing time and OBS matrix switching time to be $10 \mu \mathrm{s}$ and $2.5 \mu \mathrm{s}$, respectively. Moreover, the threshold for the burst assembly is fixed to $100 \mu \mathrm{s}$, which provides delay-sensitive mean burst lengths between 20 and 300 Kbytes in the whole range of loads under evaluation. Concerning the loss-sensitive part of the node, the BCP processing time is left to $10 \mu$ s, while the OCS matrix switching time has been increased to $1 \mathrm{~ms}$ (i.e., the slowed down dynamics of OCS allows the use of a less complex, thus cheaper switching fabric). Full wavelength conversion is assumed in all network nodes. However, no additional contention resolution strategies, such as optical buffering by means of Fiber Delay Lines (FDLs) or deflection routing, are available upon contention along the OBS one-way signalling.

For the traffic characteristics, the packet inter-arrival time at edge nodes follows a negative uniform traffic distribution and the packet size distribution is tri-modal as suggested in [[11]]. These incoming packets can be either delay-sensitive or loss-sensitive with $50 \%$ probability. Besides, they are uniformly distributed to all the remaining nodes in the network.

\section{SIMULATION RESULTS}

The performance of the previously described scenario has been evaluated by means of OMNET ++ simulations, being the main results summarized in Fig. 3. When considering the two-way reservation used for loss-sensitive traffic, the burst length is a key factor as it is tightly related to the signalling overhead. Therefore, first results shown on left graph on Fig. 3, were extracted in order to evaluate which burst length values give the best tradeoff between blocking probability (BP) and burst assembling time (during those simulations, 5 retries were allowed in case of unavailable resources at some point in reservation). It can be concluded from that graph that the BP becomes almost constant when burst sizes are greater than 40 Mbytes. This value has been therefore taken during further simulations as longer bursts would imply a similar BP value but higher assembly delays.

BP as a function of the offered load is shown in the central part of Fig. 3. It can be seen how the number of retries allowed to the loss sensitive traffic is crucial to reach the intended QoS differentiation in terns of BP. As the number of allowed retries is increased the burst loss probability is reduced. As an example, when 10 retries are allowed, the loss-sensitive traffic experiences a BP one order of magnitude lower than the delay sensitive traffic.

On the other hand, allowing a high number of retries results in high end-to-end delay values. This is studied in the right graph of Fig. 3, where it can be seen how the delay-sensitive traffic undergoes a clearly lower delay than the loss-sensitive one. It has to be highlighted that this was one of the initial objectives of this work. When considering the loss-sensitive traffic, the end-to-end delay becomes stable when the number of retries takes values higher than 5 . This implies that 10 retries can be allowed as a great improvement is obtained in terms of 
BP (see Fig. 3, centre) while the delay is only slightly affected (Fig. 3, right), because a very small number of bursts will actually need 10 retries.
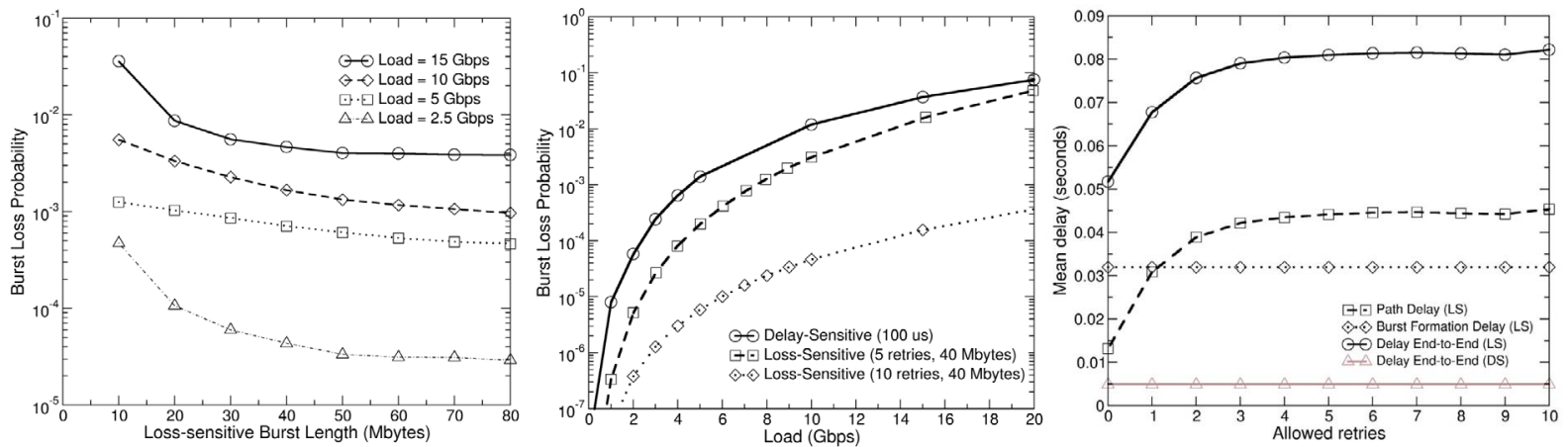

Figure 3. Simulation results: loss-sensitive burst loss probability vs. burst length (left); burst loss probability vs. offered load (center); mean end-to-end delay for delay and loss-sensitive traffic vs. allowed retries (right).

A main conclusion can finally be extracted from the presented work: it has been possible to demonstrate a clear QoS differentiation as initially expected between delay and loss-sensitive traffic types. The key parameter when trying to establish this conclusion is the number of retries allowed to the loss-sensitive traffic before being discarded.

\section{ACKNOWLEDGEMENTS}

The work reported in this paper has been partially supported by the Spanish Science Ministry through Project "Engineering Next Generation Optical Transport Networks (ENGINE)", (TEC2008-02634).

\section{REFERENCES}

[1] B. Mukherjee, "WDM optical communication networks: Progress and challenges", IEEE Journal on Selected Areas in Communications, Oct. 2000.

[2] E. Mannie, "Generalized multi-protocol label switching (GMPLS) architecture”, IETF RFC 3945, Oct. 2004.

[3] S. Yoo, "Optical packet and burst switching technologies for the future photonic Internet", Journal of Lightwave Technology, Dec. 2006.

[4] K. Dolzer, C. Gauger, J. Späth, and S. Bodamer," Evaluation of Reservation Mechanisms for Optical Burst Switching", AEÜ International Journal of Electronics and Communications, Jan. 2001.

[5] Y. Chen, C. Qiao, and X. Yu, "Optical burst switching (OBS): A new area in optical networking research", IEEE Network Magazine, May 2004.

[6] G. Zervas, R. Nejabati, D. Simeonidou, A. Tzanakaki, S. Azodolmolky, and I. Tomkos, "A hybrid optical burst/circuit switched ingress edge router for grid-enabled optical networks", in Proc. of GridNets2006, San Jose (USA), Oct. 2006.

[7] H. Vu, A. Zalesky, E. Wong, Z. Rosberg, S. Bilgrami, M. Zukerman, and R. Tucker, "Scalable performance evaluation of a hybrid optical switch", Journal of Lightwave Technology, Oct. 2005.

[8] G. Lee, B. Wydrowski, M. Zukerman, J. Choi, and C. Foh, "Performance evaluation of an optical hybrid switching system", in Proc. of Global Telecommunications Conference (GLOBECOMM 2003), San Francisco (USA), Dec. 2003.

[9] C. Xin, C. Qiao, Y. Ye, and S. Dixit, “A hybrid optical switching approach”, in Proc. of Global Telecommunications Conference (GLOBECOMM 2003), San Francisco (USA), Dec. 2003.

[10] C. Gauger, P. Kuhn, E. Breusegem, M. Pickavet, and P. Demeester, "Hybrid optical network architectures: Bringing packets and circuits together", IEEE Communications Magazine, Aug. 2006.

[11] K. Claffy, G. Miller, K. Thompson, "The nature of the beast: Recent traffic measurements from an internet backbone", in Proc. of International Networking Conference (INET), 1998. 